\title{
Closed-Incision Negative-Pressure Therapy in Obese Patients Undergoing Cesarean Delivery: A Randomized Controlled Trial
}

\author{
Ravindu P. Gunatilake, MD ${ }^{1}$ \\ Jennifer L. Thompson, MD ${ }^{1}$ \\ ${ }^{1}$ Division of Maternal-Fetal Medicine, Department of Obstetrics and \\ Gynecology, Duke University Medical Center, Durham, North Carolina \\ Am J Perinatol Rep 2017;7:e151-e157.
}

Leo R. Brancazio, MD ${ }^{1}$ Michael P. Smrtka, MD ${ }^{1}$ Beverly A. Gray, MD ${ }^{1}$ Robert Phillips Heine, $M^{1}$

\begin{abstract}
Keywords

- obesity

- cesarean

- negative pressure wound therapy

- wound infection

Background Postcesarean wound morbidity is a costly complication of cesarean delivery for which preventative strategies remain understudied.

Objective We compared surgical site occurrences (SSOs) in cesarean patients receiving closed-incision negative-pressure therapy (ciNPT) or standard-of-care (SOC) dressing.

Study Design A single-center randomized controlled trial compared ciNPT (5-7 days) to SOC dressing ( $1-2$ days) in obese women (body mass index [BMI] $\geq 35$ ), undergoing cesarean delivery between 2012 and 2014. Participants were randomized 1:1 and monitored $42 \pm 10$ days postoperatively. The primary outcome SSOs included unanticipated local inflammation, wound infection, seroma, hematoma, dehiscence, and need for surgical or antibiotic intervention.

Results Of the 92 randomized patients, 82 completed the study. ciNPT and SOC groups had similar baseline characteristics. Mean BMI was $46.5 \pm 6.5$ and no treatment-related serious adverse events. Compared with SOC, the ciNPT group had fewer SSOs (7/43 [16.3\%] vs. 2/39 [5.1\%], respectively; $p=0.16)$; significantly fewer participants with less incisional pain both at rest (39/46 [84.8\%] vs. 20/46 [43.5\%]; $p<0.001)$ and with incisional pressure (42/46 [91.3\%] vs. 25/46 [54.3\%]; $p<0.001)$; and a $30 \%$ decrease in total opioid use $(79.1$ vs. $55.9 \mathrm{mg}$ morphine equivalents, $p=0.036)$.

Conclusion A trend in SSO reduction and a statistically significant reduction in postoperative pain and narcotic use was observed in women using ciNPT.
\end{abstract}

\begin{abstract}
Address for correspondence R. Phillips Heine, MD, Division of Maternal-Fetal Medicine, Department of Obstetrics and Gynecology, Duke University Medical Center, PO Box 3967, Durham, NC 27710 (e-mail: robert.heine@duke.edu).
\end{abstract}

Cesarean delivery is the most commonly performed surgical procedure in the United States. Of the approximately 3.9 million births in the United States in 2013, more than 1.28 million (32.7\%) occurred via cesarean delivery. ${ }^{1}$ About 2 to $7 \%$ of cesarean deliveries resulted in a surgical site occurrence (SSO) leading to prolonged wound healing and postoperative pain, increased rates of secondary infection and rehospitalization, decreased patient satisfaction, and increased costs of medical

received

October 25, 2016 accepted after revision April 21, 2017
DOI https://doi.org/

10.1055/s-0037-1603956. ISSN 2157-6998. care. $^{2,3}$ Maternal obesity (BMI $\geq 30 \mathrm{~kg} / \mathrm{m}^{2}$ ) at the time of cesarean delivery is a key factor impacting postcesarean incisional morbidity. ${ }^{4,5}$ Several studies show that obese women who undergo cesarean delivery have higher rates of wound infection, seemingly independent of other known risk factors (e.g., diabetes and intrapartum intrauterine infection). ${ }^{3,5}$

Although a recent meta-analysis ${ }^{6,7}$ and prior randomized controlled trials (RCTs) ${ }^{7-10}$ of pregnant women with a range
Copyright $\odot 2017$ by Thieme Medical Publishers, Inc., 333 Seventh Avenue, New York, NY 10001, USA. Tel: +1(212) 584-4662.
License terms

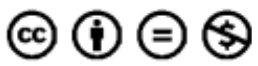


of BMIs suggest that closure with sutures is optimal to closure with staples, there are scant data regarding whether negative pressure over a closed incision might prevent postcesarean wound complications. In an RCT of high-risk lower extremity trauma patients, use of closed-incision negative-pressure therapy (ciNPT) showed decreased rates of postoperative infection and wound dehiscence. ${ }^{11}$ In an RCT of 150 obese cardiac surgery patients, applying ciNPT to sternotomy incisions significantly reduced postoperative wound infections as compared with standard-of-care (SOC) dressings. ${ }^{12}$

\section{Objective}

The primary objective of this RCT was to compare short-term clinical outcomes among obese pregnant women undergoing cesarean delivery who received ciNPT or a SOC dressing.

\section{Study Design}

We performed a single-center, postmarketing RCT at Duke University Medical Center comparing ciNPT (PREVENA Incision Management System, $\mathrm{KCI}$, an Acelity Company, San Antonio, TX), to SOC dressing. Duke Institutional Review Board approval was obtained, and active enrollment for the study occurred from 2012 to 2014; the study was registered on ClinicalTrials.gov (identifier: NCT01450631).

Pregnant women undergoing cesarean delivery were approached for study participation if they were $\geq 18$ years of age with $\mathrm{BMI} \geq 35 \mathrm{~kg} / \mathrm{m}^{2}$ at the time of delivery. Participants provided written informed consent and were enrolled either during their prenatal visits (for those undergoing planned cesarean delivery) or when they presented to the Labor \& Delivery Unit. Women with skin or systemic infections, chorioamnionitis (defined by maternal fever +1 clinical criteria), critical illness, or high-risk for anesthesia (American Society of Anesthesiologists [ASA] class P4, P5, or P6), were excluded from participation.

Immediately before cesarean delivery, all women received abdominal skin preparation with a surgical solution (Chloraprep, CareFusion, Inc., San Diego, CA) and intravenous cefazolin within 30 minutes before abdominal entry through a Pfannenstiel skin incision. Delivery and abdominal closure were performed according to standard technique with a combination of sharp and blunt dissection of the tissue planes. The peritoneum was left open, and the subcutaneous adipose tissue was closed when deemed to be $\geq 2 \mathrm{~cm}$ with chromic gut suture per institutional preference. Once the skin was reapproximated with delayed absorbable sutures, the wound was confirmed as a "clean" (Centers for Disease Control and Prevention [CDC] class 1 or 2) surgical incision, and all intraoperative inclusion/exclusion criteria were met, the patient was randomized in a 1:1 fashion to the ciNPT or SOC group. Study personnel obtained the next sequentially numbered opaque randomization envelope, which contained the randomly assigned treatment group for the participant. After being informed of the participant's assignment, trained study personnel, who included the in- vestigator/surgeon, subinvestigator/surgeon, or research nurse, applied either the ciNPT or SOC dressing to the closed incision.

A sterile, "peel-and-place," multilayer dressing (wicking fabric, reticulated foam, and adhesive) was placed over the closed incision of patients in the ciNPT group. The dressing's tubing was then attached to a compact, portable negativepressure therapy unit that delivered $125 \mathrm{~mm} \mathrm{Hg}$ of continuous pressure to the dressing and removed exudates into a disposable canister. Duration of ciNPT was $\geq 5$ to $\leq 7$ days, immediately following surgery. For women in the SOC group, Steri-Strips (3M Health Care, $1 / 2$ inch, St. Paul, MN), sterile gauze, and Tegaderm (3M Health Care, transparent film dressings [nonpenetrable barrier]) were applied to the closed surgical incision for at least 1 day and no longer than 2 days. Women receiving at least 5 days of treatment in the ciNPT group or 1 day of treatment SOC group were classified as having received treatment per protocol. All patients were discharged on postoperative day 3 or 4 in the absence of obstetrical, medical, or wound complications requiring hospitalization for management. All patients received standard wound care instructions before hospital discharge and teaching for troubleshooting the ciNPT device. Subjects randomized to the ciNPT treatment group returned for a study visit on day 6 ( \pm 1 day) to have the device removed and incision assessed.

The primary endpoint of postoperative SSOs included unanticipated local inflammatory response, prolonged drainage, fluid collection, dehiscence, and surgical site infection (SSI). SSO was counted only once, even if a patient had more than one SSO during the study period. The secondary endpoint of the study was the incidence of subjects with surgical interventions, which included antimicrobials for SSI, surgical drainage of the incision, surgical incision packing, adjunctive negative-pressure therapy, debridement, or reoperation.

Although the postoperative examiner was privy to the treatment group, a standardized wound scoring system was utilized to minimize bias. All participants were followed up postoperatively for $42 \pm 10$ days via periodic incisional assessments (postoperative days 1, 2, 6, 14, and 42) and monitored for adverse events. Surgical site assessments were performed according to a standardized wound scoring system with a score between 1 and 5 assigned for each criterion: rubor, tumor, calor, and dolor. To account for transient inflammatory responses, surgical site assessments were performed at least 1 hour following an intervention or dressing change.

Surgical site assessments included the supplementary outcomes of incisional pain scores at rest and with pressure on the closed incision, as measured by the Wong-Baker Faces Scale. A pain score greater than or equal to 2 was selected as the threshold for a positive outcome, as the decision to seek narcotic analgesics are subjective and variable with exception to a pain score of zero. Any occurrences of SSO were managed according to institutional practice (e.g., antibiotics and reexploration) as needed. Additional data collected included: antibiotic use; suture line assessment; and medication use. All narcotics and analgesic therapies (such as inpatient doses of 
acetaminophen, nonsteroidal anti-inflammatory drugs [NSAID], and opioid narcotics [e.g., oxycodone, hydrocodone]) used during participants' postoperative hospital stays were obtained from the medication record by the investigator after the study was completed. As narcotic use was not standardized per study protocol, narcotic usage was converted to morphine equivalents.

The study was originally designed to compare the mean number of SSOs in each treatment group. The mean and standard deviation of the number of SSOs for ciNPT and SOC were assumed to be 1.0 (1.0) and 2.5 (2.5), respectively. The study would have $80 \%$ power to detect this difference with 30 subjects enrolled in each group. Per protocol, an interim analysis of the first 30 subjects was performed. Based on the interim analysis of the primary outcome, it was determined that the SSO count was no longer relevant and analysis would be done on the incidence rate. Based on the interim analysis results, SSO rates of $6 \%$ ciNPT and $31 \%$ SOC, enrollment was increased to 46 participants in each treatment group.

Statistical analysis was performed using SAS Version 9.2, (SAS Institute Inc., Cary, NC). All statistical tests were twosided, with type I error rate of $5 \%$. Baseline characteristics were defined before or immediately after cesarean delivery. All categorical variables were summarized as frequencies and percentages. Continuous data were expressed as mean, standard deviation, median, and range (minimum, maximum) unless otherwise noted. Analyses of proportions, chisquare test, or Fisher's exact test were used for all compar- isons between the ciNPT and SOC groups. No adjustments were made to $p$ values for multiple comparisons. The Student's t-test was used for the subsequent analysis of analgesic use.

\section{Results}

Of the 101 women consented and assessed for study eligibility, 9 were considered screen failures and were not included in the study. As shown in the CONSORT ${ }^{13}$ patient flow diagram (-Fig. 1), a total of 92 women were randomized to either the ciNPT or SOC groups (46 in each group).

The intent-to-treat (ITT) population ( $n=92$ ) included all randomized participants in the treatment arm to which they were randomized. The per-protocol (PP) population $(n=82)$ consisted of 39 ciNPT and 43 SOC dressing participants who completed the study within the required visit windows and had no protocol deviations. Final analysis of study results for SSO included all $82 \mathrm{PP}$ participants. In the seven women who did not complete the study, no SSO events were noted before their discontinuation. It is unknown if an SSO event occurred within $42 \pm 10$ days. Final analysis of study results for participant-reported pain at the incision site was based on the ITT population, which included all 92 randomized women (46 women per group).

Demographic and operative characteristics were calculated from the ITT population and were similar between the

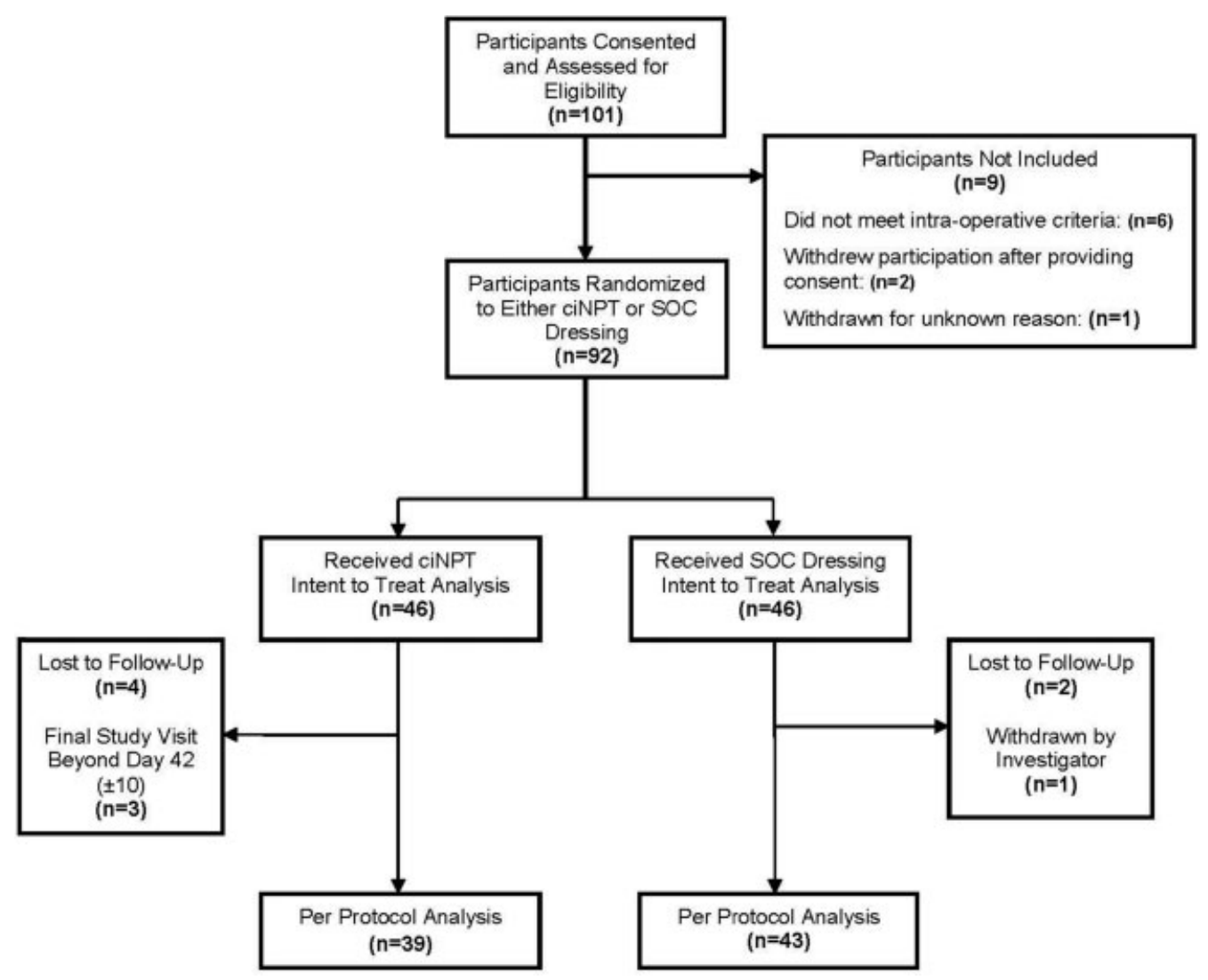

Fig. 1 CONSORT study population diagram. 
Table 1 Demographic and incisional characteristics in the ciNPT, SOC, and overall treatment groups calculated from the ITT population

\begin{tabular}{|c|c|c|c|}
\hline & $\begin{array}{l}\text { ciNPT } \\
(N=46)\end{array}$ & $\begin{array}{l}\text { SOC } \\
(N=46)\end{array}$ & $\begin{array}{l}\text { Overall (ITT) } \\
(N=92)\end{array}$ \\
\hline Age $(y)$, mean (SD) & $30.4(5.7)$ & $29.7(5)$ & $30.0(5.3)$ \\
\hline BMI $\left(\mathrm{kg} / \mathrm{m}^{2}\right)$, mean (SD) & $46.3(7.3)$ & $46.8(5.6)$ & $46.5(6.5)$ \\
\hline BMI $\left(\mathrm{kg} / \mathrm{m}^{2}\right)$, median (minimum, maximum) & $46.4(35.7,60.8)$ & $45.4(38.9,60.8)$ & $45.8(35.7,60.8)$ \\
\hline \multicolumn{4}{|l|}{ Race } \\
\hline American Indian, $n(\%)$ & $0(0 \%)$ & $1(2.2 \%)$ & $1(1.1 \%)$ \\
\hline African American, $n$ (\%) & $29(63.0 \%)$ & $35(76.1 \%)$ & $64(69.6 \%)$ \\
\hline Caucasian, $n(\%)$ & $17(37.0 \%)$ & $10(21.7 \%)$ & $27(29.3 \%)$ \\
\hline Hispanic or Latino, $n(\%)$ & $3(6.5 \%)$ & $2(4.3 \%)$ & $5(5.4 \%)$ \\
\hline \multicolumn{4}{|l|}{ Repeat or primary cesarean } \\
\hline Primary, $n(\%)$ & $11(23.9 \%)$ & $15(32.6 \%)$ & $26(28.3 \%)$ \\
\hline Repeat, $n(\%)$ & $35(76.1 \%)$ & $31(67.4 \%)$ & $66(71.7 \%)$ \\
\hline Diabetes, $n(\%)$ & $8(17.4 \%)$ & $8(17.4 \%)$ & $16(17.4 \%)$ \\
\hline Gestational age (wk), mean (SD) & $38.1(2.0)$ & $37.9(2.0)$ & $38.0(2.0)$ \\
\hline Incision length $(\mathrm{cm})$ & 16.3 & 16.3 & 16.3 \\
\hline Pfannenstiel incisions (percentile) & 100.0 & 100.0 & 100.0 \\
\hline
\end{tabular}

Abbreviations: ciNPT, closed-incision negative-pressure therapy; ITT, intent-to-treat; SD, standard deviation; SOC, standard-of-care dressing.

treatment groups ( $\mathbf{-}$ Table $\mathbf{1}$ ). All women received preoperative antibiotics (cefazolin $2-4 \mathrm{~g}$ based on body weight) within 30 minutes of the incision. None of the women underwent incisional drain placement, anticoagulant therapy within 24 hours of cesarean delivery or cesarean hysterectomy.

Women who completed the study (PP, $n=82$ ) had an overall SSO incidence of $11 \%$ (-Table 2). Compared with women in the SOC group, those in the ciNPT group had a $63 \%$ relative reduction in SSOs (7/43 [16.3\%] vs. 2/39 [5.1\%], $p=0.16)$ and fewer SSIs (4/43 [9.3\%] vs. $1 / 39$ [2.6\%)], $p=0.36$ ) (-Fig. 2). Women in the SOC group were also more likely to have a dehiscence (5/43 [11.6\%] vs. $1 / 39$ [2.6\%], $p=0.20)$ and require surgical incisional intervention $(6 / 43$
[14\%] vs. $1 / 39,2.6 \%], p=0.11$ ); however, these differences did not reach statistical significance.

Overall participant-reported pain scores using the WongBaker Faces Scale with a value of $>2$ (any pain) postoperatively were evaluated between the treatment groups. In the ITT population ( $n=92$ ), the ciNPT group, compared with the SOC group, had significant reductions in both pain at rest (20/46 [43.5\%] vs. 39/46 [84.8\%], respectively; $p<0.001)$ ( - Fig. 3) and pain with pressure applied (25/46 [54.3\%] vs. 42/46 [91.3\%], $p<0.001$ ) (-Fig. 4).

Regarding postcesarean analgesia requirements, in the ciNPT group, compared with the SOC group, total narcotic use was reduced by $30 \%$ (55.9 vs. 79.1 ) parenteral morphine equivalents; $p=0.036$ ). When comparing women in the ciNPT group to those in the SOC group, there was no

Table 2 Breakdown of surgical site occurrences

\begin{tabular}{|l|l|l|l|}
\hline & $\begin{array}{l}\text { ciNPT } \\
(\boldsymbol{n}=39)\end{array}$ & $\begin{array}{l}\text { SOC } \\
(\boldsymbol{n}=43)\end{array}$ & $\boldsymbol{p}$ Value \\
\hline Surgical site occurrence, any & $2(5.1 \%)$ & $7(16.3 \%)$ & 0.16 \\
\hline Unanticipated local inflammatory response & $0(0 \%)$ & $0(0 \%)$ & \\
\hline Prolonged drainage & $0(0 \%)$ & $0(0 \%)$ & 0.36 \\
\hline Fluid collection (seroma, hematoma, abscess) & $1(2.6 \%)$ & $4(9.3 \%)$ & 0.20 \\
\hline Dehiscence & $1(2.6 \%)$ & $5(11.6 \%)$ & 0.11 \\
\hline Surgical incision intervention, any & $1(2.6 \%)$ & $6(14.0 \%)$ & \\
\hline
\end{tabular}

Abbreviations: ciNPT, closed-incision negative-pressure therapy; SOC, standard-of-care dressing. 


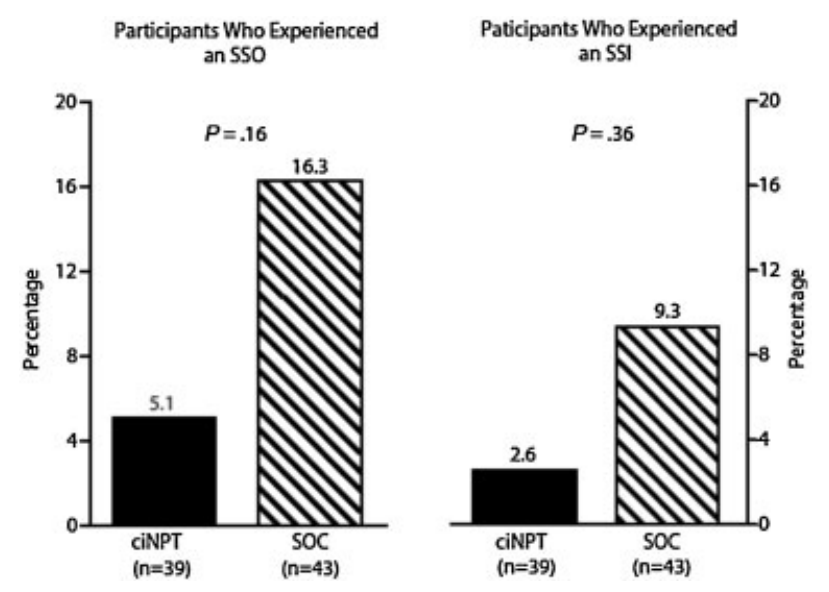

Fig. 2 Wound complication rates. The percentage of participants in the ciNPT and SOC groups who had any SSO and those who developed a SSI calculated from the per-protocol population $(n=82)$. SSO, surgical site occurrence; SSI, surgical site infection; ciNPT, closedincision negative-pressure therapy; SOC, standard-of-care dressing.

statistically significant difference for total acetaminophen use (6,882 vs. $6,924 \mathrm{mg} ; p=0.47)$ and total NSAID use (1.65 vs. 1.51 maximum daily use equivalents; $p=0.87$ ) ( - Fig. 5).

\section{Conclusion}

Postcesarean wound complications, specifically in the extremely obese pregnant population, remain a major issue in modern obstetrics. In this study of ciNPT versus SOC dressing for incision management in an obese population undergoing cesarean delivery, we demonstrated a trend toward reduction in incisional wound complications and a statistically significant reduction in postoperative pain and narcotic use.

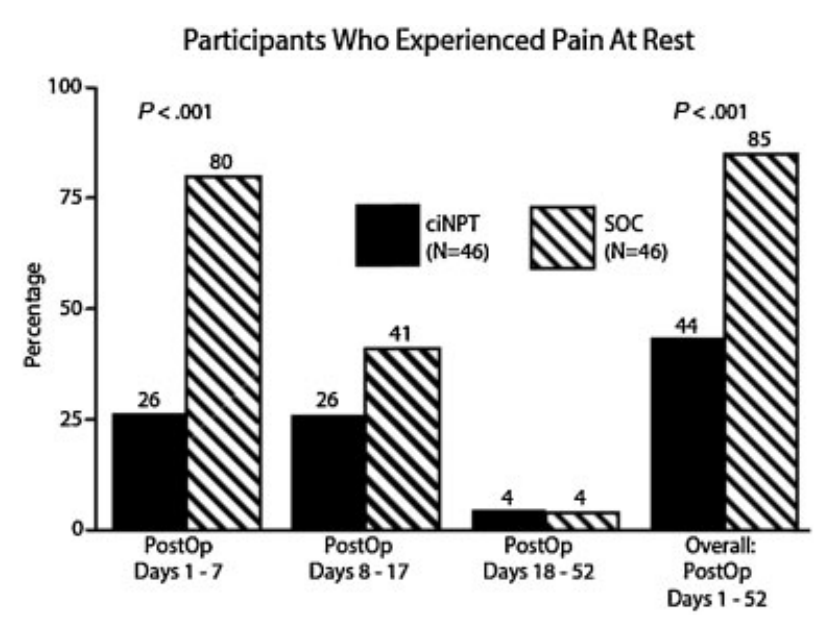

Fig. 3 Participant-perceived pain scores at rest. Percentage of participants who reported pain at rest with a value of $>2$ (any pain) using the Wong-Baker Faces Scale, calculated from the intention-totreat population ( $n=92$ ). ciNPT, closed-incision negative-pressure therapy; SOC, standard-of-care dressing.

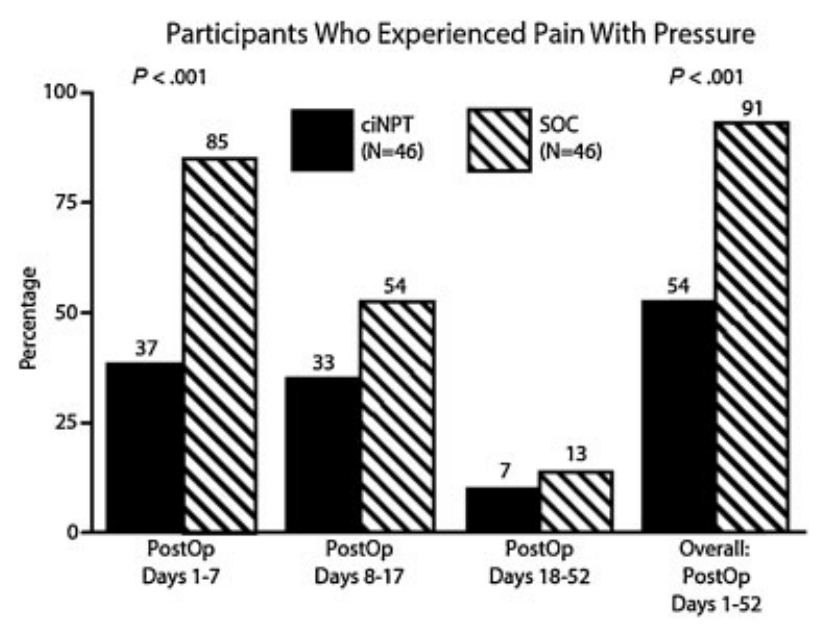

Fig. 4 Participant-perceived pain scores with pressure. Percentage of participants who reported pain with pressure applied to a value of $>2$ (any pain) using the Wong-Baker Faces Scale, calculated from the intention-to-treat population $(n=92)$. ciNPT, closed-incision negative-pressure therapy; SOC, standard-of-care dressing.

The study population was appropriate for the clinical question at hand, that is, women at relatively high-risk for SSOs at baseline with a BMI $>35 \mathrm{~kg} / \mathrm{m}^{2}$ at the time of cesarean delivery and without evidence of systemic infection. However, after the planned interim analysis and sample size adjustment, the study remained underpowered for the primary outcome due to an unexpected reduction in SSOs. Since there were no institutional changes to cesarean technique or postoperative follow-up care, factors aside from treatment may have affected this postinterim reduction in SSO incidence. One possible factor was the lack of laboring women in our study after an interim analysis. Other factors include the possibility of a "Hawthorne effect," in which the process of observation alone results in a reduction in the primary outcome distinct from the intervention itself.

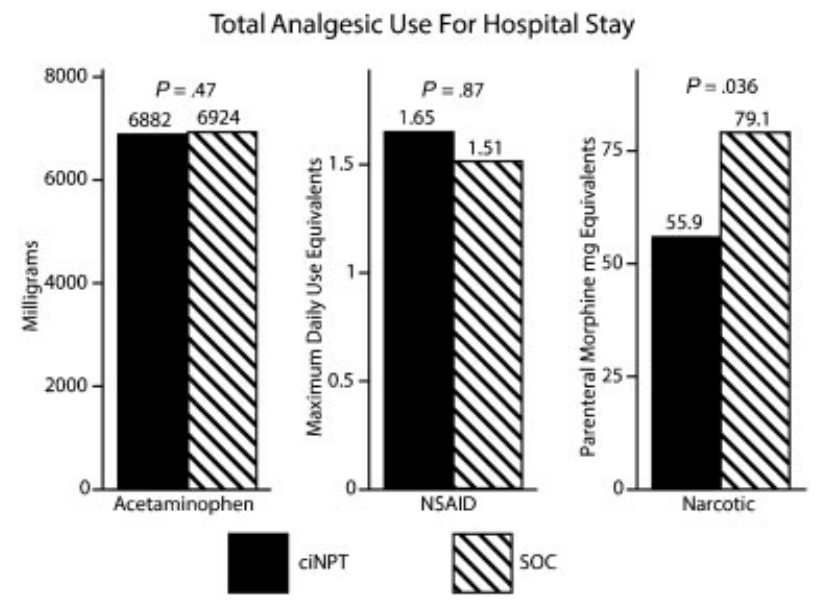

Fig. 5 Total analgesic use for hospital stay. Cumulative total inpatient use of acetaminophen, NSAID, and narcotic opioid medications expressed as milligrams, maximum daily use equivalents, and parenteral morphine mg equivalents, respectively. ciNPT, closed-incision negative-pressure therapy; NSAID, nonsteroidal anti-inflammatory; SOC, standard-of-care dressing. 
Moreover, the study did not control for primary versus repeat cesarean incisions though it is unlikely to be a significant factor given that repeat cesarean has not been demonstrated to be an independent risk factor for postcesarean wound morbidity previously. Although obesity is a major risk factor for infection, it is likely that other cofactors such as labor or chorioamnionitis play a significant role in the development of wound complications.

The trend toward a reduction of SSOs with ciNPT is clinically relevant, given that SSIs were a component of the primary outcome and carry substantial costs to the individual patient and health care system. A typical hospital readmission costs approximately $\$ 6,600$, for a postcesarean SSO. ${ }^{2,14}$ The present study adds to the limited body of evidence regarding postoperative ciNPT following cesarean. In a nonselective retrospective case-control study, Gibbs showed no reduction in SSIs, when 103 patients with ciNPT were compared with 867 historical controls after controlling for obesity and diabetes. ${ }^{15}$ In contrast, a prospective casecontrol study by Swift et al showed a significant $70 \%$ reduction in wound complications among a high-risk group of 110 postcesarean women treated with ciNPT versus 209 historical controls. ${ }^{16}$ Similarly, Mark and colleagues had no postoperative infections in a group of 21 obese patients versus a $10 \%$ rate in 42 historical controls. ${ }^{17}$ In our RCT, we also demonstrated a reduction in wound complications, which is consistent with these recent studies in high-risk populations. The results of this study are consistent with other data that suggest that ciNPT may reduce wound complications in high-risk women. However, further studies are needed to determine whether the same benefit applies to low-risk women undergoing cesarean.

Although potentially subject to bias, the significant reduction in postoperative incisional pain, which was supported by a decrease in narcotic use among women in the ciNPT group, has significant implications for postpartum and postoperative pain management. Prior studies of postcesarean analgesia techniques (e.g., patient-controlled analgesia) have noted that ineffective pain relief may exacerbate the risk for thromboembolism via immobility as well as reduce mother-infant bonding and breastfeeding, which are complications of particular clinical relevance in an obese obstetrical population. ${ }^{18,19}$ More recently, studies evaluating medical and psychosocial risk factors in patients with opioid dependence have identified the antecedent use of postoperative opioid analgesia as a potential "gateway" to dependence. ${ }^{18}$ If further research confirms or expands on our finding of a significant reduction in postoperative pain and total opioid narcotic intake, the use of ciNPT may be considered for its ancillary benefits in postcesarean women.

The obesity epidemic and its management are, and will likely continue to be, a central focus of current obstetric care for the foreseeable future. Treatment strategies such as ciNPT are needed to reduce cost and associated morbidity of wound complications. We acknowledge the need for further RCTs with adequate power to examine the impact of ciNPT in riskdiverse obstetric populations. It is imperative that such studies account for additional benefits beyond wound complications, such as postoperative pain management, narcotic utilization, and patient satisfaction.

\section{Note}

Study results were presented at: the 41 st Annual Meeting of the Infectious Diseases Society for Obstetrics and Gynecology, August 7-9, 2014; Stowe, VT.

\section{Funding}

The study was sponsored by KCI, an Acelity Company, San Antonio, TX.

\section{Disclosure}

To the author's knowledge, no conflict of interest, either financial or other, exists.

\section{Acknowledgments}

The authors would like to acknowledge Kristin Weaver, MS, CRC at the Duke University Medical Center for study coordination and data management.

\section{References}

1 Centers for Disease Control and Prevention. Births-Method of delivery. Available at: http://www.cdc.gov/nchs/fastats/delivery. htm. Accessed October 07, 2015

2 Edwards JR, Peterson KD, Mu Y, et al. National Healthcare Safety Network (NHSN) report: data summary for 2006 through 2008, issued December 2009. Am J Infect Control 2009;37(10): 783-805

3 Perlow JH, Morgan MA, Montgomery D, Towers CV, Porto M. Perinatal outcome in pregnancy complicated by massive obesity. Am J Obstet Gynecol 1992;167(4 Pt 1):958-962

4 Alanis MC, Villers MS, Law TL, Steadman EM, Robinson CJ. Complications of cesarean delivery in the massively obese parturient. Am J Obstet Gynecol 2010;203(03):271.e1-271.e7

5 Olsen MA, Butler AM, Willers DM, Devkota P, Gross GA, Fraser VJ. Risk factors for surgical site infection after low transverse cesarean section. Infect Control Hosp Epidemiol 2008;29(06):477-484, discussion 485-486

6 Tuuli MG, Rampersad RM, Carbone JF, Stamilio D, Macones GA Odibo AO. Staples compared with subcuticular suture for skin closure after cesarean delivery: a systematic review and metaanalysis. Obstet Gynecol 2011;117(03):682-690

7 Mackeen AD, Khalifeh A, Fleisher J, et al; CROSS Consortium. Suture compared with staple skin closure after cesarean delivery: a randomized controlled trial. Obstet Gynecol 2014;123(06):1169-1175

8 Rousseau JA, Girard K, Turcot-Lemay L, Thomas N. A randomized study comparing skin closure in cesarean sections: staples vs subcuticular sutures. Am J Obstet Gynecol 2009;200(03):265.e1-265.e4

9 Basha SL, Rochon ML, Quiñones JN, Coassolo KM, Rust OA, Smulian JC. Randomized controlled trial of wound complication rates of subcuticular suture vs staples for skin closure at cesarean delivery. Am J Obstet Gynecol 2010;203(03):285.e1-285.e8

10 Figueroa D, Jauk VC, Szychowski JM, et al. Surgical staples compared with subcuticular suture for skin closure after cesarean delivery: a randomized controlled trial. Obstet Gynecol 2013; 121(01):33-38 
11 Stannard JP, Volgas DA, McGwin G III, et al. Incisional negative pressure wound therapy after high-risk lower extremity fractures. J Orthop Trauma 2012;26(01):37-42

12 Grauhan O, Navasardyan A, Hofmann M, Müller P, Stein J, Hetzer R. Prevention of poststernotomy wound infections in obese patients by negative pressure wound therapy. J Thorac Cardiovasc Surg 2013;145(05):1387-1392

13 Moher D, Hopewell S, Schulz KF, et al; CONSORT. CONSORT 2010 explanation and elaboration: updated guidelines for reporting parallel group randomised trials. Int J Surg 2012; 10(01):28-55

14 Agency for Healthcare Research Quality. HCUPnet. Healthcare Cost and Utilization Project. Available at: http://hcupnet.ahrq.gov/. Accessed October 7, 2015

15 Gibbs C, Orth T, Gerkovich M, Heitmann E, Parrish M, Lu G. Traditional dressing compared with an external negative pressure system in preventing wound complications. Obstet Gynecol 2014; 123:145S

16 Swift SH, Zimmerman MB, Hardy-Fairbanks AJ. Effect of singleuse negative pressure wound therapy on postcesarean infections and wound complications for high-risk patients. J Reprod Med 2015;60(5-6):211-218

17 Mark KS, Alger L, Terplan M. Incisional negative pressure therapy to prevent wound complications following cesarean section in morbidly obese women: a pilot study. Surg Innov 2014;21(04):345-349

18 Gadsden J, Hart S, Santos AC. Post-cesarean delivery analgesia. Anesth Analg 2005;101(5, Suppl):S62-S69

19 Woods AB, Crist B, Kowalewski S, Carroll J, Warren J, Robertson J. A cross-sectional analysis of the effect of patient-controlled epidural analgesia versus patient controlled analgesia on postcesarean pain and breastfeeding. J Obstet Gynecol Neonatal Nurs 2012;41(03):339-346 